[Aus der kgl. Universitäts-Augenklinik zu Halle a. S. (Dir.: Prof. Dr. E.v.Hip pel.)]

\title{
Ein Beitrag zur Kenntnis der Neubildungen am Limbus corneae.
}

Von

Dr. med. Kurt Heilbrun, Assistenzarzt der Klinik.

Mit Taf. XVIII, Fig. 1-4, und 6 Figuren im Text.

Der Limbus corneae ist der bevorzugte Sitz für Neubildungen am Auge. Ich hatte Gelegenheit 4 Limbustumoren zu beobachten und anatomisch zu untersuchen, die verschiedene Typen der von dem Epithel ausgehenden Neubildungen darstellen: 1. eine anatomisch benigne tumorähnliche rezidivierende Epithelhyperplasie, 2. ein Papillom mit Ubergreifen auf die Hornhaut (das Präparat überliess mir in liebenswürdiger Weise Herr Prof. v. Hippel aus seiner Sammlung); 3. und 4. zพ̀ Ci Carcinome.

Die benigne Epithelhyperplasie am Limbus, wie sie bei dem Patienten beobachtet wurde, ist bisher noch nicht beschrieben worden, sie soll deshalb auch in ausführlicher Weise mitgeteilt werden. Vielleicht ist das, was Hocquard mit "Plaques épidermiques" bezeichnet, etwas ähnliches. Die Arbeit war mir nicht im Original zugängig. Sie ist citiert nach Lagrange (Encyclopédie franç.). W. T. Lisler and W. I. Handcock demonstrierten Präparate von epithelialen Plaques am 28. VII. 1903 in der British medical Association, über die Art dieser Präparate konnte ich gleichfalls nichts Genaueres erfabren und muss es daher offen lassen, ob die Befunde dem unsrigen ähnlich sind.

\section{Krankengeschichte.}

Der jetzt 50 Jahre alte Eisenbahnsehaffner Fr. Dr. steht seit dem 16. III. 07 bei uns in Behandlung.

Im Sommer 06 hatte Patient auf dem reehten Auge eine Augenentzündung, die von hereingeflogener Flugasehe herrühren sollte, die Patient 
nicht zu entfernen vermochte. Das Avge rötete sich, ohne äztliche Behandlung jedoch ging die Entzündung in ungefähr 8 Tagen unter warmea Unschlägen und Aufstreichen einer Salbe zurück. Anfang 07 machten Bekannte den Patienten darauf aufmerksam, dass sich am rechten Auge etwas Abnormes bilde. Anfang März 07 ging er zum Kassenarat, der ibn der Augenllinik zu Halle uberwies.

Patient ist nie ernstlieh angenkrank gewesen, hat stets gat geselen und gibt an, dass sich die jetzt zeigende Neubildung zuerst am Homhautrande gebildet und allmählich nach der Pupille über die Hornhaut vorgeschoben habe.

Status: 16. III. 07. Gesunder kräftiger Mann. Aus dem Tränensack ist kein Sekret ansdribekbar. Die Conjunctiva palpebarum ist etwas hyperämisch, sonst normal.

Ein grosser Teil der Cornea des reehten Auges wird im temporalen

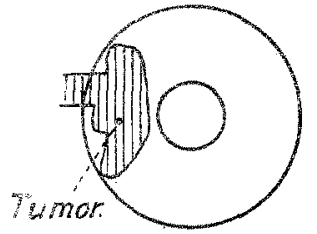

Fig. 1. Teile ron einem grauweissen, wenig erhabenen Tumor eingenommen, der eine gestichelte Oberfläche hat und von zahlreichen Gefässen durehzogen wird. Ein Zapien erstreckt sich nach dem Hornhautrande hin. Er geht rom oberen Drittel des Tumors ab, in ihm verlaufen zahlreiche Gefässe.

Die Pupille reagiert anf Licht, keine Synechien, sonst normaler Befund.

V. R. $+0,5 D . S=6 \int_{5}$ L. $+0,5 D . S=5$.

Am 19. III. 07 wurde der Tumor oberfachlich abgetragen, die WundAlache wurde gründlich abgeschabt (Geheimrat Schmidt-Rimpler),

Am 28. III. 07 wude Patient entlassen. Die Stelle, an der der Tumor sass, ist epithelisiert, etwas vnregelmässig, halb durchscheinend.

V. $+1,0$ D. $S=5 / 5$.

Eine anatomische Untersuchung des Tumors fehlt.

Nicht ganz ein Jahr darauf, am 19. II. 08, stellte sich Patient mit einem Rezidiv wieder vor.

Die Conjunctiva bulbi ist in ihrem temporalen Teile leicht injiziert, der Tumor sitzt wieder anf der alten Stelle, doch ausschliesslich auf cornealem Gebiet. $\mathrm{Er}_{\mathrm{r}}$ ist etwa $1 \mathrm{~mm}$ hoch, $4 \mathrm{~mm}$ breit, $6,25 \mathrm{~mm}$ lang. Seine Oberfläche ist zerkluftet, rollkommen weiss. Der Tumor zeigt keine Gefässe.

I. Rezidiv.

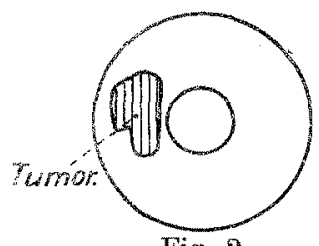

Fig. 2. V. $+1,0$ D. $S=5 /$. . Auge sonst 0. B. Am 22. II. 08 wird der Tumor mit dem Graefe-Messer in mehreren Stücken abgetragen. An der Basis des Tumors zeigen sich mehrere kleine Gefässe. Der Wundboden wird mit Lapis geätzt (Geheimrat Schmidt-Rimpler).

Der abgetragene Tumor ist nicht anatomisch untersucht worden. Im Verlazfe der Heilung trat ein zentrales Hornhautinfiltrat ein, das unter friedlicher Therapie zuriekging. Am 13. III. 08 wird Patient als geheilt entlassen. An der Stelle des Tumors findet sich eine Macula mit unregelmässiger Oberfläche.

$$
\text { V. }+1,0 D . S=\left.5\right|_{10}
$$


Ein am 28.V. 08 von Geheimrat Schmidt-Rimpler erstattetes Gutachten glaubt einen ätiologischen Zusammenhang zwischen oben erwähntem Trauma und Tumor immerhin nicht ausschliessen zu können.

Am 27. VI. 08 ist die Narbe glatt.

V. $+1,0 D \cdot S=5 \mid$

Am 20. XI. 08 ist die Cornealnarbe noch glatt, aber anschliessend an die Narbe findet sich auf der Conjunctiva oben innen eine etwas verdickte, umschriebene, leicht verschiebliche, sulzige Partie, die den Eindruck eines beginnenden Rezidives macht. $V .+1,0 D . S=5 / 7,5$.

Die weiteren Skizzen sollen die flächenhafte Ausbreitung der Wucherung dartun. Der Tumor breitete sich ziemlich schnell über einen grossen Teil der Conjunctiva und Cornea aus:

Der Visus betrug am 11. X. $09+1,0 D C$ $+2,0 D \mathrm{cyl} . S=5 / 10^{\text {. }}$

Am 22. XI. 09 erfolgte wiederum Aufnahme in die Klinik.

Das Auge ist blass, nur an der Stelle des Tumors besteht conjunctivale und eiliare Injektion. Der Tumor nimmt den oberen Teil der Hornhant und der angrenzenden Conjunctiva ein. Er hat sulzigen Cha-

II. Rezidiv.

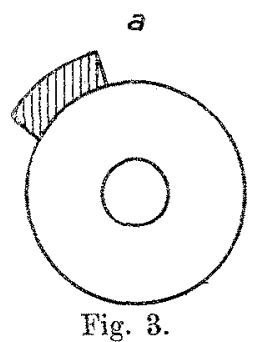

(20. XI. 08.) rakter und ist nur wenig exhaben. Der conjunctivale Teil ist von massenhaften neugebildeten Gefässen durehsetzt, auch der corneale Teil hat solche Gefässe. Masse: Breite $19 \mathrm{~mm}$, Höhe $8 \mathrm{~mm}$.

$b$

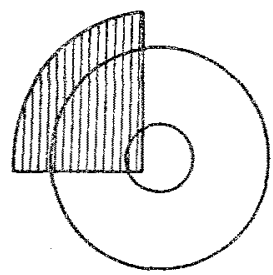

Fig. 4.

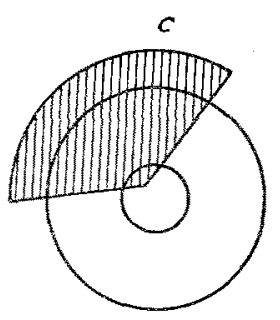

Fig. 5.

(13. II. 09.)

(11. X. 09.)

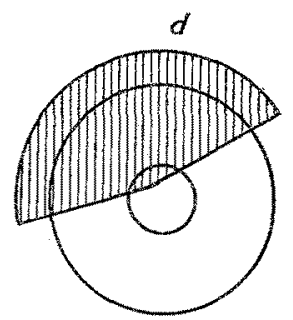

Fig. 6.

(22. XI. 09.)

In dem nasalen und unteren Abschnitt der Hornhaut finden sich einige feine Maculae.

Pupille reagiert prompt. Conjunctiven und inneres Ange sind völiig normal. V. $+1,0 . D=+1,5 \mathrm{D}$ cyl. $s=5 / 15$.

Die Diagnose wurde kliniseh auf maligne Neubildung gestellt und zwar wahrscheinlich Epitheliom, dafür sprachen: 1, der Sitz am Corneallimbus, der ja der Lieblingssitz maligner Tumoren am Auge ist, 2. das schnelle und wiederholte Rezidivieren, und 3, das relativ schnelle Wachstum.

Limbusverändernngen, wie sie bei Frühjahrskatarrh forkommen, waren von vornherein auszuschliessen, da der Prozess einseitig war, die Conjunctiven absolut keine Erscheinungen boten, die für Frühjahrskatarrh sprachen, und der ganze Verlauf unbeeinflusst von der Jahreszeit vor sich ging.

Am 23. XI. 09 wurde zunächst eine Probeexcision aus dem mitt- 
leren Teile des Tumors vorgenommen wegen der Frage der eventuellen Enucleation.

Fixieren in absolutem Alkohol. Einbetten in Paraffin, HämatoxylinEosinfürbung.

Es war nor ein sebr kleines Stüekchen. Ws zeigte sich, dass es sich um eine gewaltige Epithelwucherung handelte. Der glatten Unterlage aus Bindegewebe sassen zunächst cylindrische Epithelzellen in mehrfacher Lage auf, dann kamen weitere massenhafte Epithellagen von unregelmässiger Konfiguration. Die obersten Epithellagen zeigten Degenerationen des Protoplasma, Vakuolenbildungen, schlechte Färbbarkeit der Kerne und Lockerung der Kittsubstanz. Hin und wieder fand sich eine Epitheiperle. Ein destruierendes Wachstum war nirgends nachweishar.

Nach diesem Befunde handelte es sich demnach nicht um ein Carcinom. Da aber das zur Untorsuchung gekommene Stick nur sehr klein war, so war natiirlich nicht auszuschliessen, dass an andern Stellen infiltratives Wachstum bestünde.

Am 26. XI. 09 wurde der Tumor möglichst im Gesunden in 2 Teilen abgetragen (Prof. v. Hippel).

Der Tumor lässt sich von der Unterlage gut abpräparieren. Die Hornhaut unterhalb des Tumors ist durehsichtig, die Homhautwunde wird dureh herbeigezogene Bindehautlappen gedeckt.

Die Heilung erfolgt reaktionslos.

Im 23. XII. 09 wird Patient als geheilt entlassen, der Bindehautlappen deckt den oberen Homhantteil glatt, ist im ganzen dünn geworden, nur nasal zeigt er noch etwas Wulstung. Der Visus beträgt $5 / 10$ mit kombiniertem Cylinderglas. - Patient stellt sich in 4 wöchentlichen Intervallen vor und ist bis jetzt (29. VIII. 10) rezidivfrei. Die Conjunctiva deckt glatt die Hornhautwunde.

Anatomische Untersuchung: Der Tumor wurde in $4 \%$ Formalinlösung fixiert, in der aufsteigenden Alkoholreihe gehärtet und in Paraffin eingebettet. Die Präparate wurden mit Hämatoxylin-Eosin gefärbt.

Die bindegewebige Grundlage des Tumors ist nicht gewuchert, doch auffallend gefässreich. Von den im conjunctivalen Bindegewebe liegenden Gefässen zieben ab und zu Ästchen in die Epithellagen hinein. Die Bowmansche Membran ist im cornealen Teile der Wucherung völlig intakt. Die zunächst gelegenen Teile des Hornhautparenchyms zeigen reichlich gefüllte Gefässe bís dicht an die B ow man sche Membran heran. Die gewucherten Epithelien heben sich im conjunctivalen Teile vom Conjunctivalepithel in scharfer Linie ab (vgl. Taf. XVIII, Fig. 1: Übersichssbild) ${ }^{1}$ ). Die Epithelschieht ist im conjunetivalen und cornealen Teile ungefahr gleich dick. Es finden sich $30-40$ Epithellagen bubereinander. Die unteren Zellagen sind vorwiegend eylindrisch, nach oben hin werden sie von unregelmässiger Begrenzung. Vorwiegend in der äusseren Hälfte der Epithelmassen zeigen sich Degenerationserscheinungen: Homogenisierung des Protoplasma, Vakuolenbildung, schlechte Färbbarkeit der Kerne, Lockerung der Kittsubstanz. Hin

1) Die mikrophotographischen Aufnahmen machte Herr Prof. v. Hippel, an dieser Stelle sei ihm dafür mein herzlichster Dank ansgedrückt. 
und wieder findet sich eine Epithelperle (vgl. Taf. XVIII, Fig. 2: Epithel bei starker Vergrösserung photographiert). Zwischen den Epithelzellen liegen im conjunctivalen Teile des Tumors Gefässe in mässiger Menge. Stellenweise reichen sie bis zur Mitte der Epithelwucherung. Im cornealen Teile fehlen Gefässe zwischen den Epithellagen.

Die anatomische Diagnose lautet nach dem mitgeteilten Befunde auf hochgradige Hyperplasie des Epithels der Cornea und angrenzenden Conjunctiva.

Anatomisch handelte es sich um einen benignen Prozess, da jede Spur eines destruktiven, infiltrativen Wachstumeś fehlt.

Es sei noch einmal hervorgehoben, dass eine Beteiligung des Bindegewebes nicht bestand.

Klinisch können wir nicht von einer Gutartigkeit des Prozesses sprechen, da in relativ kurzer Zeit 2 Rezidive der Abtragung folgten, der Prozess sich ziemlich schnell ausbreitete (vgl, die oben mitgeteilten Skizzen) und der Visus infolgedessen erheblich sank.

Dass das geringe Trauma, das der Patient angibt, auslösend gewirkt haben könnte, hält Geheimrat Schmidt-Rimpler immerhin für möglich, wie er sich in seinem Gutachten ausdrückte, doch ist zu bedenken, dass ja eine ziemlich lange Zeit zwischen dem geringfügigen Trauma: Hineinfliegen von Flugasche, und dem Auftreten des Tumors verging (ungefähr ${ }_{1 / 2} \mathrm{Jahr}$ ).

Hervorzuheben ist ferner noch, dass die Conjunctiva palpebrarum völlig normal ist, nicht die geringsten Einlagerungen zeigt, denn es wäre immerhin denkbar, dass irgendwelche Einlagerungen in die Conjunctiva infolge Reizung Hyperplasie des Epithels hervorrufẹn könnten, wie es ja an andern Körperstellen infolge chronischer Reizeinwirkungen vorkommt.

Wie schon oben erwähnt wurde, scheinen bisher nur von zwei Autoren gleiche Befunde erhoben worden zu sein. Nahestehende Befunde veröffentlichen noch drei Autoren. In diesen Fällen besteht jedoch eine Mitbeteiligung des Bindegewebes und z. T. Bildung rudimentärer Papillen. Während der eine (Magnus) seinen Fall ohne weiteres den Papillomen zuzählt, tun es die andern zwar auch, doch unter Hinweis auf das abweichende Verhalten gegenüber den typischen Papillomen. Diese Fälle stehen in der Mitte zwischen dem oben mitgeteilten und den Papillomen, auf die wir weiter unten noch $\mathrm{zu}$ sprechen kommen werden.

Zunächst soll noch kurz auf die Beobachtungen eingegangen werden. 
Der erste Beind warde yon Magn us (Klin. Monatsbl.f. Augenheilk. 1887. S. 384) mitgeteilt.

Es handelt sich um einen 12 jährigen Knaben. Am rechten inneren Hornhautrande besteht eine leicht prominierende graurötiche $4 \mathrm{~mm}$ lange, $2 \mathrm{~mm}$ breite Wuchernng, die sich ein wenig anf die Hornhaut hinaulschiebt, hier grauweisslieh, speckig erseheinend. Einzelne Gefügse ziehen aus der Conjunctiva hinein. Am äusseren findet sich eine ähnliche, jedoch erheblich kleinere Wucherung, am oberen eine $9 \mathrm{~mm}$ lange, $1 \mathrm{~mm}$ breite. Die Conjunetiva zeigt keine paihologischen Veränderungen. Das linke Ange ist röllig normal.

Auf Jodkali und Zinktropfon sehienen sich zunächst die Nerbildungen zu verkleinern. Bald zeigte sich jedoch wieder ein vermehrtes Wachstum. Nun wurde die Wucherung abgetragen. Kurze Zeit darauf trat ein Rezidir mit ziemlich schnellem Wachstum ein. Am linken Auge traten jetzt aueh ähnliche Wucherungen auf. Verf. trug den Tumor am inneren Rande der Homhant ab und untersuehte iln mikroskopiseh.

Die mikroskopisehe Untersuchung ergab: Auf einer zellreichen Verdiekung der Bindegewebssehicht, die reichlich vaskularisiert war, sass eine Epithelschicht, die sich dureh eine wellige Linie scharf gegen die Unterlage absetzte. Die Epithellage war stark verdickt, an sinzelnen Stellen stärker als an andern. Stellenweise erhob sich das Epithel papillenartigg (ich möehte her gleich betonen, dass die Papillen nur ans Epithel bestanden).

Es konnten Kernteilungsfiguren in den vergrösserten Kernen nachgewiesen werden. Manche Zellen zeigten Protoplasmanetzwerk; in den mittleren Lagen fanden sich Riff- oder Stachelzellen.

Auf Grund des Vorkommens von Epithelpapillen stellt Magnus die Diagnose auf Papilloma corneae.

Dass von einem Papillom keine Rede sein kann, erhellt daraus, dass keine Beteiligung des Bindegewebes an den Papillen bestand. Wenn es sich in dem Falle Magnus nicht um Wucherungen bei Frühjahrskatarrh handelt, wie Fuchs (cit, nach Velhagen) und Velhagen einwenden, so steht dieser Fall dem von mir mitgeteilten immerhin sehr nahe, wenn auch eine Wucherung des Bindegewebes besteht.

Über 2 hierher gehörige Fälle berichtet noch Mathieu 1906 im Arch. f. Augenbeilk. Bd. LV. S. 223:

Fall I: Der unteren äusseren Partie der Hornhaut sitzt ein Tumor auf, der $1-2$ mm über den Limbns hinaus auf die Conjunctiva bulbi übergreift. Der Tumor hat fleiselige Konsistenz, sieht intensiv weiss ans und hat leicht höckexige Oberfläche.

Mikroskopischer Befund: „Die Grundlage des Tumors besteht aus einem fein faserigen, leicht gewellten Bindegewebe, weiches nur an wenigen Stellen riehtige kurze, aber ziemlieh breite Papillenstöeke bildet, darüber erheben sich mächtige, vielfach zerklüftete papillomatöse Wucherungen, welche 
nur aus Epithelzellen, die in der Hauptsache undeutlich begrenzt sind, bestehen. Thre Form ist in der Tiefe mehr rundlich, gegen die Oberfläche des Tumors flacher. Ein dentlicher Plattenzellenüberzug findet sich meist nur an der Spitze der Papillen, während in der Tiefe der Einsenkungen die oberflächlichen Zellen meist keine Plattenzellen, sondern versehiedengestaltig sind, rundlich, öfters auch annähernd cylindrisch und dann senkrecbt zur Oberfläche stehend. Namentlich in den tieferen Schichten haben die Zellen öfters ein scholliges Aussehen. Becherzellen kommen nur vereinzelt vor. Das Epithel setzt sich scharf von dem Bindegewebe ab, hat nirgends Tendenz in die Tiefe zu wuchern, zeigt keine Mitosen."

Fall II: Früher behandelt wegen Scleritis und kleiner Wucherung an der: Corneaskleralgrenze des rechten Auges, die abgetragen aber nicht anatornisch untersucht worden war.

Aufnahmebefund: Im unteren nasalen Quadranten der Hornhaut des rechten Auges sitzt ein glatter, wulstiger, weicher Tumor, der die Corneaskleralgrenze überschreitet und fast bis zur unteren Übergangsspalte reicht. Eine ähnliche ungefähr stecknadelkopfgrosse Wucherung sitzt am oberen Hornhautrand. Der Tumor wurde abgetragen.

Mikroskopischer Befund: "Die Geschwulst besteht in der Hauptsache aus einer kompakten Masse epithelialer Zellen, welche sich gegen die unveränderte Grundlage mit einer welligen linie scharf absetzt. Nur an wenigen Stellen tritt eine papilläre Anordnung hervor. Der Grundstock solcher Papillen besteht aus dünnwandigen Blutgefässen, die von spärlichen Bindegewebszügen umgeben sind. Die die Papillen begleitenden Zellen haben in ihrer basalen, dem Grundstock aufsitzenden Schicht vielfach Oylinderepithelcharakter und zeichnen sich durch intensivste Kernfärbung aus, während die weiter nach oben liegenden Zellen des Überzugs mehr blass, grösser and sehollig werden. Dies Verhalten wiederholt sich regelmässig da, wo Papillen oder Andeutungen davon verhanden sind. Im allgemeinen sind die Zellen zienlich gleichmässig, die Zeichnung ruhig, nennenswerte Polymorphie sowie Mitosen sind nicht zu sehen. An wenigen Stellen finden sich in den obersten Schichten Hornperlen and zwar da, wo auch die Zellmassen im ganzen verhornt sind und die Oberfläche stellenweise etwas gefurcht ist. In der Hauptsache ist dio Oberfäche, abgesehen ron den Stellen, an welchen die oben erwähnten Papillen vorkommen, eine glatte, leicht gewellte."

Der ausführlich mitgeteilte anatomische Befund von Mathieus Fällen zeigt, dass das Hervortretendste die Wucherung des Epithels war. Im zweiten Falle war das Bindegewebe nicht gewuchert, wohl dagegen im ersten. Papillenbildungen sind in beiden Fällen in mässigen Mengen, aber nur rudimentär entwickelt vorhanden. Das Epithel uberzieht die Bindegewebslage in scharf begrenzter welliger Linie. Im ersten Falle fanden sich Fpithelpapillen, wie im Falle Magnus. Der zweite Fall steht dem unsrigen noch näher als der erste, da eine Wucherung des Bindegewebes fehlt und die gewucherte Epithelschicht eine glatte Oberfläche zeigt. 
Präparate, die den Befunden von Magnus und Mathien nahestehen, demonstrierte Natanson jun. in der Moskauer augenärztlichen Gesellschaft am 17. IV.07. Genaueres ist dem Referate in den Klinischen Monatsblättern für Augenheilkunde 1907. Bd. XLV, 1. S. 577 nicht zu entnehmen.

Fassen wir noch einmal das Wichtigste über die bisher mitgeteilten Befunde zusammen:

1. Am Limbus corneae kommen anatomisch benigne Epithelhyperplasien vor, die klinisch den Eindruck einer malignen. Neubildung machen können infolge ihrer Rezidivfähigkeit und ihres flächenhaften Wachstumes.

2. Diesen Epithelhyperplasien steht eine Gruppe von Wucherungen nahe, die von Magnus, Mathieu und Natanson beschrieben wurden, die hauptsächlich eine Wucherung des Epithels zeigen, das sich scharf gegen die bindegewebige Unterlage absetzt. Die bindegewebige Unterlage ist in diesen Fällen z. T. mitgewuchert und zeigt z. T. vereinzelte, im anatomischen Bilde zurücktretende Bildung von Papillen. Auch in diesen Fällen besteht klinisch Rezidivfähigkeit und flächenhaft sich ausbreitendes Wachstum.

Ich komme nunmehr zur Beschreibung eines Falles von Papillom des Limbus. Saemisch hat im Handbuch in der Bearbeitung der Erkrankungen der Conjunctiva die Tumoren des Limbus mit abgehandelt, ich habe infolgedessen die Literatur erst ron dem Jahre 1904 an weiter zusammengestellt, sie findet sich in dem beigegebenen Literaturverzeichnis. Eine übersichtliche Zusammenstellung der Literatur bis zum Jahre 1904 verdanken wir noch Velhagen in Vossius' Sammlung zwangloser Abhandlungen aus dem Gebiete der Augenheilkunde vom Jahre 1904.

Bis zum Jahre 1904 wurden nach Velhagens Zusammenstellungen 95 Fälle von Papillomen der Conjunctiva publiziert.

Von 74 Fällen liess sich feststellen, ob sie solitär oder multipel vorkamen, 56 waren solitär, 18 multipel.

Von den solitären Geschwülsten sassen 18 auf dem Limbus corneae, 13 auf der Plica semilunaris, bzw. Karunkel, 8 auf der Conjunctiva bulbi, 5 auf den Übergangsfalten, 2 am äusseren Lidwinkel.

Die multipel aufgetretenen Papillome fanden sich 9 mal über die Conjunctiva zerstreut, 4 mal am Limbus, 3 mal an der Karunkel, 2 mal auf der Lidbindehaut.

Das Papillom, über das ich berichten werde, sass am Limbus corneae. Eine Krankengeschichte steht mir nicht zur Verfügung. 
Wie die beigegebene Mikrophotographie (Taf. XVIII, Fig. 3) erkennen lässt, reicht es von der Übergangsfalte bis über die Mitte der Cornea.

Das Präparat ist in Celloidin eingebettẹt and nach van Gieson gefärbt.

Wenden wir uns gleich dem histologischen Befunde zu: Das subconjunctivale Gewebe im Bereiche des Tumors zeigt reichliche Vaskularisation, stellenweise kleinzellige Infiltration. Von diesem Gewebe aus erheben sich feine Bindegewebszüge, die in ihrem Innern Gefässe führen. Diese Bindegewebszüge verästeln sich nach der Peripherie des Tumors hin immer feiner. Alle diese Züge sind von gewaltigen Epithelmassen bekleidet, die da, wo sie der bindegewebigen Grundlage aufsitzen, cylindrisch sind, sehr bald aber einen polymorphen Charakter annehmen. Stellenweise glaubt man nur Epithelmassen vor sich zu haben, da eine Sonderung der einzelnen Papillenstöcke im mikroskopischen Bilde häufig nicht möglich ist und das bindegewebige Gerïst in seinen feinen Auslänfern ganz zurïcktritt. An dem cornealen Teile sind die obersten Sehichten der Substantia propria reich vaskularisiert und kleinzellig infiltriert. Für das bindegewebige Geriist und das Epithel der Papillen gilt dasselbe wie vom conjunctivalen Teile des Tumors. Interessant ist noch die Partie, an der das Papillom gegen die übrige Hormhaut sich absetzt, hier ist die Oberfläche des Epithels unregelmässig, verschieden dick, jedenfalls in Proliferation, und hin und wieder sieht man in das Epithel hinein einen dicken bindegewebigen Spross sich vorsehieben.

Diese Art der Papillome, bei denen das Epithel die Hauptmasse des Tumors ausmacht, ist, wie man den Zusammenstellungen ron Velhagen entnehmen kann, bereits beschrieben. Es kommt aber auch das Gegenteil vor, dass nur 3-4 Epithellagen einer dickeren bindegewebigen Lage aufsitzen.

Das Papillom ist anatomisch eine völlig benigne Geschwulst; klinisch muss auch sie anders bewertet werden, da sie 1. nach der Abtragung sebr häufig rezidiviert, 2. sich der Fläche nach ausbreitet und dadurch den Visus, speziell bei Sitz in der Gegend der Cornea schädigen kann, und 3. mitunter zu recht erheblichen, ja lebensgefährlichen Blutungen führt.

Schliesslich ist zu bedenken, dass nicht allzu selten aus einem Papillom ein Carcinom sich entwickelt. So fand Velhagen unter 90 Fällen $4 \mathrm{mal}$ sicheren Utbergang in Carcinom.

Das Papillom kommt in jedem Lebensalter vor und ist nicht, wie von manchen behauptet wird, im wesentlichen nur eine Erkrankung des jüngeren Alters zum Unterschiede vom Carcinom, das hauptsächlich in höherem Alter auftreten soll.

Wir kommen damit zum letzten Abschnitte der Arbeit, zur Mitteilung zweier Fälle von primärem Carcinom. 
Fall I. Dem 20 jährigen Landwirt A. R. war vor angeblich 15 yahen ungelöschter Kalk ins linke Auge gespritzt. Im Anschluss daran soll sich oin rötliehes Enötchen auf dem skleralen Teile der Bindehaut gebildet haben und zwar temporalwärts rom Hornhautrande, von inm dureh eine weisse Zone getrennt. Das Knötchen soll sich ganz allmählich vergrössert haben. Vor einem Jahre soll der Hornhautrand erreicht worden sein, dann soll die Geschwulst auf die Hornhaut selbst übergegriffen haben. Wesentiche Besehwerden haben nie bestanden.

Patient ist völlig gesund.

Temporal yom Hormhantrande ist die Conjunctiva bulbi von einer hellroter Aachen Geschwulst ron ovaler Form eingenommen. Die Masse betragen 12:9 mm. Nach oben erstreekt sich dieselbe ungefähr bis zum vertikalen Meridian der Hornhaut, nach unten hin reicht sie nur wenig bis unterhalb des hurizontalen. An der Oberfläche der Geschwulst sieht man ein nicht besonders enges Netz sehr feiner Gefässe, die am stärksten besonders oben anssen sind, gegen den Rand der Geschwulst hin in die Tiefe ziehen und hier noch eine Strecke weit durch das transparente Gewebe der Geschwulst gesehen werden können. Die Oberfläche der Geschwulst ist im ganzen glatt. Man hat nieht den Eindruck einer stärkeren Epithelverdickung. In der ganzen Ausdehnung der Gesehwulst ist der Limbus corneae orgriffen und sieht graurot ans. Ungefähr entspredend dem horizontalen Meridian schiebt aich eine geringe Verdickung vom Limbus her auf die Hornhaut hinüber.

Der Bulbus ist sonst normal. Patient hat $5 / 5$ Sehschärfe.

Die präaurikniäre Lymphdrüse ist nicht geschwollen.

Operation and Verlauf: Der corneale Teil des Tumors wird mit der Lanze umsclnitten and unterminiert. Der sklerale Teil wird mit der Schere von der normalen Conjunctiva abgetrennt. Es entsteht eine ziemlich starke Blutung. Der Tumor lâsst sich dann ohne grössere Sohwierigkeiten in toto exstirpieren. Die Hornhaut wird, soweit sie vom Tumor eingenommen war, kauterisiert. Sie ist noch ziemlich durchsichtig; dann wird die gesunde Conjunctiva von oben und unten her darïber vernäht, so dass der Hornhautdefekt völlig von Conjunctiva gedeckt ist (Prof. v. Hippel).

Der Wundverlauf ist völlig glatt and bereits nach 7 Tagen kann der Patient entlassen werden.

Eine klinische Diagnose über die Art des Tumors zu stellen, war nicht möglich, er sah äusserlich dem oben beschriebenen Falle von Hyperplasie des Epithels völig ähnlich und bot anatomisch doch ein ganz anderes Bild.

Anatomische Untersuchung: Der Tumor wurde in der modifizierten Zenkerschen Flüssigkeit fixiert, in Jod-Alkohol gehärtet, in Paraffin eingebettet; die Schnitte wurden nach van Gieson gefärbt.

Das mikroskopische Bild ist ein ungewöhnliches. Das nicht verdickte Oberflächenepithel wuchert infiltrativ in die Tiefe, z. T. in soliden Epithelsäulen, z. T. in schlauchartigen Epithelmassen: ein Hohlraum ist umgeben von einer mehrfachen Lage kubiseher Epithelzellen. Zwischen den einzelnen Epithelmassen liegt Bindegewebe in mässiger Menge (vgl. Taf, XVII, Fig. 4). 
Fall II: Der 60 Jahre alte Arbeiter Karl B. hat als Kind wiederholt Angenentzïndungen durchgemacht, die letzte vor 9-10 Jahren. Seit dieser Zeit war das Auge ab und zu einmal rot, seit 2 Jahren bemerkte er am rechten Ange ein Gewächs, das an der temporalen Seite begonnen und sich allmählich über die Hornhaut vorgeschoben haben soll. Seit 6 Wochen hat er Schmerzen am rechten Auge. Er wurde lange Zeit von seinem Arzt mit Augensalbe ohne Erfolg behandelt.

Das linke Auge ist normal. Der Mann ist sonst völlig gesund.

Auf der Hornhaut des rechten Auges findet sich den grössten Teil derselben einnehmend eine höckerige, über den Limbus hinausragende Geschwulst, zu der sehr reichliche Blutgefässe verlaufen. Die Ränder sind gezackt und uneben. Von der Hornhaut selbst sieht man nur noch einen kleinen nasalen Sektor, der leicht parenchymatös getrübt, dabei aber noch gut durchsichtig ist. Zwisehen der relativ intakten Zone der Hornhaut und dem Tumor findet sich nasal vom Zentrum eine stark getrübte Partie, die wohl noch der Hornhautsubstanz angehört und zu der ein Gefäss verläuft. Die Iris ist bräunlich, leicht verwaschen, von der Pupille ist nur ein kleiner Teil zu sehen. Die Pupillarreaktion ist nicht mit Sicherheit auslösbar. Vom Hintergrund ist kein Reflex zu erhalten. Die Pupille erweitert sich nicht auf Atropin. Visus $=$ Handbewegungen in $1 \mathrm{~m}$.

Die Diagnose lautete auf Carcinoma corneae.

Der Bulbus wurde enucleiert, in modifizierter Zenkerscher Lösung fixiert, in Jod-Alkohol gehärtet, in Celloidin eingebettet. Die Präparate wurden nach van Gieson gefärbt.

Der anatomische Betund zeigt folgendes:

Auf der einen Seite am Limbus corneae beginnend und auf der andern Seite fast bis zam Limbus reichend ist das Epithel der Cornea in dicken Massen in die Tiefe gewuchert, die Bowmansehe Membran ist ganz zerstört. Die soliden Zellmassen, die nur hin und wieder etwas Bindegewebe zwischen sich lassen, sind durch den grössten Teil der Substantia propria gewachsen. Die Descemetsche Membran legt sich stellenweise in Falten. Am Epithel fällt die reichliche Bildung von Epithelkernen auf. Das sich zwischen die Carcinomzellen schiebende Bindegewebe ist stark infiltriert, besonders aber auch das subconjunctivale Gewebe auf der Seite, wo das Carcinom den limbus nock nicht erreicht hat.

Im Fall II handelte es sich um ein typisches, vom Limbus ausgegangenes Hornhautcarcinom bei einem alten Manne, welches das gewöbnliche Verhalten: flächenhafte Ausbreitung bei geringer Wucherung in die Tiefe, zeigt.

In Fall $\mathrm{I}$ handelte es sich um ein Carcinom bei einem jungen Individuum. Der 20jährige Patient hat vor 15 Jahren im Anschluss an ein Trauma ein rötliches Knötchen auf der Conjunctiva bulbi bekommen, aas sich allmählich vergrössert hat. Die Bildung des Carcinoms scheint demnach im Alter ron 5 Jahren begonnen zu haben. Es sind nur wenige Fälle von Carcinomen am Limbus in so frühem Alter bekannt. 
Saemisch stellt folgende Fälle von Frühauftreten zusammen: Der jüngste Patient war 20 Monate alt, dann folgen solche im Alter von $6,12,13,19,27,35,37$ Jahren. Gewöhnlich tritt ja das Limbuscarcinom erst nach vollendetem 40 . Lebensjahre auf.

Weiter beachtenswert ist noch im Fall I die eigenartige Struktur des Carcinoms, das z. T. aus massiven Zellsäulén, z. T. aus Epithelschläuchen besteht. Die Bildung von Epithelschläuchen ist im ganzen selten. Ein schlauchartiges Epitheliom der Skleralbindehaut, das auf die Karunkel übergrift, publizierte $1898 \mathrm{~K}$ opff (Epithelioma volumineux de la conjonctive bulbaire. Recueil d'opht. p. 264. Ref. Michels Jahresbericht 1898).

Klassifizieren wir zum Schluss noch einmal kurz die hier abgehandelten, vom Epithel ausgehenden Prozesse der Limbusgegend. Am Limbus corneae kommen von derartigen Wucherungen vor:

1. Benigne, tumoräbnliche, rezidivierende Epithelhyperplasien.

2. Epithelhyperplasien, bei denen auch die bindegewebige Grundlage eine Verdickung erfahren hat und wo stellenweise Papillen angedeutet sind (Übergangsformen zu den Papillomen).

3. Typische Papillome.

4. Primäre Carcinome (die sekundären Carcinome fanden keine Berücksichtigung).

\section{Literaturverzeichnis.}

(Die Literatur wurde fom Jahre 1904 an zusammengestellt; soweit altere Arbeiten eingehender benutzt wurden, sind sie mit angefuhrt worden.)

Saemisch, Krankheiten der Conjunctiva im Handb. v. Graefe-Saemisch. Lagrange et Valude, Eneyclopédie française d'Ophtalmologie. $V$.

Panas, Maladies des yeux.

Norris and Oliver, System of Diseases of the eye.

Epithelhyperplasie des Limbus.

1881. Hocquard eit. nach Lagrange, Encyclop. franc. d'opht. V. p. 1140.

1903. Lister, W. T., u. Handcock, W. I., Epitheliale Plaques der Conjunctiva. British Medieal Assoe. 28. Juli, Ref, Klin. Monatsbl. Bd. XLII, 1. S. 96.

Papillome der Conjunctiva und Cornea.

1887. Magnus, Ein Fall von Papilloma conjunetivae. Klin. Monatsbl.f. Augenheilk. S. 384.

1904. Faith, Papilloma of the conjunctiva. Ophth. Record. p. 223.

Heilmüller, Papilloma of the limbus conjunctiva. Journ. of the Amer. med. Assoc. 31. Okt. 1903.

Johnston, Papilloma of the conjunctiva, Opht. Record. p. 195.

Pascheff, Papillome limbo-connéen. Clinique Opht. 10. Mai.

Velhagen, Uber die Papillombildung auf der Conjunctiva. Vossius Sammlung Heft 7 .

1905. Cosmettatos, Papillome de la conjunctiva bulbaire ayant envahi la comé. Annales d'oculist. T. CXXXIII. p. 39. 
Dupay-Dutemps, Papillomes du sac lacrymal et papillomes de la conjonctive. (Présentation des preparations. Société d'opht. de Paris.) Recueil d'opht. p. 37.

Fischer, G., Über einen Fall von Papillom der Hornhaut. Inaug.-Diss. Tübingen.

Johnston, Another case of papilloma of the conjunctiva. Ophth. Record.p. 217.

Pascheff, Papilloma limbi conjunctivae. Ophth. Hosp. Reports. XVT. Part III. p. 370.

Schulek, Papillome der Hornhaut und des Limbus. Ber. über d. 1. Vers. d. ungar. ophth. Ges. in Budapest.

1906. Lauber, H., Beitrag zur Erkenntnis der Entstehung der Hornhautpapillome. Zeitschr. f. Augenheilk. Bd. XVI.

Ischreyt, vgl. Literaturangabe über Carcinome.

Mathieu, Beitrag zu den Tumoren des Limbus. Arch. f. Augenheilk. Bd. LV. S. 223.

Noiszewski, Papillom der Bindehaut übergehend in ein Epitheliom und ein Endotheliom (polnisch). Post. oculist. August.

1907. Loeb, Papilloma of the caruncle (St. Louis med. Society). Ophth. Record. p. 386.

Monthus, Papillome de la conjonctive bulbaire. (Société d'opht. de Paris.) Recueil d'opht. p. 183.

Natanson, Papilläres Epitheliom der Conj. bulbi. Moskauer Aug.-Ges. 17. IV. 1907.

1908. Alt, On eonjunetival cysts and papillomata. Americ. Journ. of Ophth. p. 292. Faith, Papilloma of the conjunctiva, with report of a case successful treated with the $\mathrm{X}$ ray. Journ. of Ophth. and Oto-Laryng. p. 130 and (Chicago Ophth. Society.) Ophth. Record. p. 247.

Gutmann, $\mathrm{Zu}$ den Geschwülsten der Caruncula lacrymalis. Zeitschr. $f$. Augenheilk. Bd. XIX. S. 16. (3 Fälle von Papillom der Conjunetiva, Epithel aus 3-4 Zellagen bestehend, kubisch.)

Hil bert, Zur Kenntnis der polypoiden Geschwülste der Bindehant. Zentralbl. f. prakt. Augenheilk. S. 139. (Ohne anat. Befund.)

Moissonnier, Papillome de la cornée. Arch. d'opht. XXVIII, p. 686. Natans on, Ein Fall von Epithelioma papillare conj. bulbi. (Sitz. d. Ophth. Ges. in Moskau. 17. April 1907.) Westn. Ophth. Bd. XXV. S, 125.

Smith, Fugene, Case of papilloma of the cornea. Ref. Klin. Monatsbl. f. Augenheilk. Bd. XLVI, 2. S.96. (Wenig bindegewebige Stützsubstanz, sehr viel Epithel.)

1909. Jou dine, Papillome de la cornée. Annal. d'ocul, 1910. p. 143.

Wiegmann, Ein Fall von epibulbärem Papillom. Klin. Monatsbl. f. Augenheilk. Beil.-Heft 1909. S. 109.

1910. Jacovides, Hornhautpapillom. Ber. d. ophth. Ges. in Ägypten. Jahrg. 1908/09. Ref. Klin. Monatsbl. f. Augenheilk. 1910. S. 373.

Reis, Utber diffuse Papillomatose der Hornhaut. Zeitschr. f. Augenheilk. Bd. XXIV, 2. S. 129.

\section{Carcinome der Conjunctiva und Cornea.}

1904. Danseau, Épithéliome de la conjonctive et de la cornée. Montpellier méd, p. 3522. Ref. Révue générale d’opht. 1905. p. 110.

Kau f $\operatorname{mann}$, E., Eigenartiger Fall von Careinom am Limbus corneae. Ophth. Klin. S.9. (Ulcerierter Plattenepithelkrebs mit reichlichen Perlkugeln und Neigung zu Blutungen.)

Neuhann, Beitrag zur Kenntnis der Hornhautcarcinome. Inaug.-Diss. Leipzig. (5 Fälle.)

Tello, Un caso epithelioma primitivo, de oftalm. hisp.-americ. p. 686. (Nur klinisch beobachtet.)

1905. Ayres, C. M. A., and Ayres, W. M. L., Epibulbär papillo-epitheliom. Americ. Journ. of Ophth. p. 242. (Ursprünglich Papillom der Cornea-skleralgrenze, schliesslich Carcinom, die ganze Orbita ansfüllend.) Blohm, Zur Kasuistik der malignen Limbustumoren. Inang.-Diss. Rostock.

ఛ. Graefe's Arehiv fur Ophthalmologie. LXXYII. 3. 
Gasparini, Di un caso di carcinoma primitivo della cornea. Annal. di Ophth. XXXIV. p. 95.

Ischreyt, Über epibulbäre Carcinome. Zeitschr. \&. Augenheilk. Bd. XII. S. 430 . (2 Fälle.)

Ray, Tumors of the conjunctiva and cornea. Transact. of the Americ. Ophth. Society. Fortym first Meeting Vol. X. p. 571. (1. papilläres Epitheliom ansgehend yon einer Hornhautnarbe, 2. Hornhantearcinom, das in die Vorderkammer gewuchert ist.)

Ray u. Vorhoff, Tumors of the conjunctiva and cornea. Ophthalmologie octobre 1905. (1. papilläres Hornhautepithelion un ein Geschwïr sich entwickelnd, 2. carcinomatöses Dermoid des Limbus.)

Schulek, Carcinoma villosum keratodes des Limbus. Ber. über d. Vers. d. ungar. ophth. Ges. in Budapest.

1906. de Berardini, Primäres Epitheliom der Hornhant. Vers. d. ital, ophth. Ges. 11. XI, 06 in Rom. - Abtragung, Überpflanzung von Kaninchenhornhaus. Rezidiv, Wiederholung desselben Verfahrens, nach $1 \mathrm{Jahr}$ rezidivfrei. Ref. IXlin. Monatsbl. f. Augenheilk. Bd. XLV, 1. S. 406.

Borsello, Su di un caso periteliona della conjunctiva bulbare. Annali di Ottalm. XXXV. p. 272.

Cosmettatos, Epithélioma épibulbare. Arch. d'opht. XXVI. p. 448.

Fleischer, Uber eine vlceröse Form des Carcinoma epibulbare. Ver. d. Württemberger Augenärzte. Ophth. Klin. Nr. 13. (Ohne Tumorbildung, in Form eines Geschwitrs verlaufend, vom Limbus auf Hornbaut und Conjunctiva übergreifend.)

Goldzieher, M. u. W., Über die malignen epibulbaren Geschwülste. Szemészeti Capok. Nr. 4.

Ischreyt, Klinische und anatomische Studien an Augengeschwülsten. Berlin, Karger. (1. Ce. d. Conj. palp., 2. Ce. der Karunkel, 3. Papillom der Plica semilun.)

Petit, Epithelioma de la caroncule. Annal. d'ocul. T. CXXXVI. p. 37.

1907. v. Barley, Epibulbäres Epitheliom. Zeitschr. A. A ugenheilk. Bd.XVII. S. 86 . Baudry, Un cas d'épithéliome primitif de la conjonctive bulbaire. Le Nord med. Nr. 308.

Debève, Tote sur un cas d'epithelioma primitif de la conjonctive bulbaire. Arch. d'opht. XXVII. p. 478. (In der Gegend des Schlemmschen Kanales bereits in die Tiefe wuchernd.

Frugiuele, Sulla prognosi dell' epithelioma epibulbare. (XIX. Riunione dell'Assoc. of. Ital.) Il Progresso Oftalm. p. 186. (4 Fälle: 3ेmal Abtragung, 1 mal Enucleation.) Ref. Michels Jahresber. S. 596.

Jennings, A case of epithelioma of the ocular conjunctiva of unusual size. Annal. of Ophth. April 1906.

Münz, Über ein carcinomatös' entartetes Papillom der Tränenkarunkel. Inaug.-Diss. Jena. (Derselbe Fall demonstriert von Wagenmann in der med-naturwiss. Ges. Jena. Deutsche med. Wochenschr. S. 1318.)

Puccioni, Epitheliome della congiuntiva et della cornea. XIX. Congr. dell' Assoc. Oft. Ital. Parma. (Ganze Hornhant war eingenommen. Drucksteigerung. Enucleation. - Der Vater, wegen Hornhautcare, enucleiert, war an allgemeiner Carcínose eingegangen.)

Salaset Ribas, Epithelioma epibulbar del ojo izquierdo. Arch. de Oft. hisp.-americ. Januar n. Maí.

1908. Aurand, Epithelioma de la caroncule. Revue générale d'opht. p. 145. (Melanotisches Epitheliom.)

Barmettler, Contributo allo studio della morfologia e structura dei tumori epibulbari; un caso di epithelioma initiale della congiuntiva bulbare. Giornale internaz. delle szienze med, 15. febbrajo.

Boulai, Le carcinome mélanique. Son point de départ règle son traitement. Clinique Opht. p. 323.

Crampton, Epithelioma of the sclero-cornea limbus. Section on Ophth. College of Physic of Philadelphia. Febr, 1907. (Innerhalb 16 Jahren sich entwickelnd; $\% / 3$ der Hornhaut, ein Teil der Conjunetiva war ergriffen.) 
Frugiuele, Contribution à l'étude des tumeurs péribulbaires. Arch. d'opht. XXVIII. p. 490.

Napp, Gesehwülste des Limbus corneae. Zeitschr. f. Augenheilk, Bd.XX. S. 515. (1. Cc, vom Bindehautepithel ausgegangen, 2. Carcinoma sarcomatodes.)

de Schweinitz and Hollway, Epithelioma of the irner angle of the eye cured by X rays. (Section on Ophth. of Physic of Philadelphia.) Ophth. Record. p. 93.

Stalberg, Demonstration von Präparaten von einem Fall von Epithelioma corneae. Sitzungsber. d. schwed. augenärztl. Ver. Hygieia. S. 892. (Nach vergeb licher Behandlung mit Röntgenstrahlen, Exstirpation des Tumors.) Strachow, Tumor des Auges. Sitzungsber. d. Ophth. Ges. in Noskau. Westn. Ophth. Bd. XXV. S. 119. (Breitbasiger, auf Hornhaut und Sklera sitzender, wahrscheinlich maligner Tumor. - Ohne anat. Befund.)

1909. Delord et Reevel. Sur deux cas d'epithelioma de la conjonctive bulbair. Annal. d'oculistes, p. 432. (1. Cc. zwischen Karunkel and Hornhaut, 2. Limbuscc. und Krebsnest im Corpus ciliare.)

Napp, Präparate eines Limbustumor. (Carcinom oder alveoläres Sarkom). Berl. Ophth. Ges. Ref. Klin. Monatsbl. f. Augenheilk. 1910. S. 208.

Snegirew, Ber. d. Sitz. d. Mosk. Augenarzte-Ges. 24. II. 1909. (Epithelkrebs 4 mal rezidiviert, nach Abtragung immer kauterisiert. Fine kleine Phlyktänen ähnliche Partie am Limbus.

1910. Bergmeister, Rudolf. Ophth. Ges. in Wien. 22. XIr. 1909. Ref. Arch. f. A ugenheilk. S. 225. (Epitheliom, die Hornhant total einnehmend. Zugleich besteht ein Hautkrebs des Gesichts.)

Steiner, Zwei Geschwülste der Caruncula lacrymalis. Zentralbl. f. prakt. Augenheilk. Juni 1910. (1. Adenom, 2. Retentionscyste.)

\section{Erklärung der Abbildungen auf Taf. XVII, Fig. 1-4.}

Fig. 1. Epithelhyperplasie des Limbus corneae. Übersichtsbild.

Fig. 2. Fpithelhyperplasie des Limbus corneae bei starker Vergrösserung.

Fig. 3. Papillom des Limbus. Übersichtsbild.

Fig. 4. Eigenartiges Limbuscarcinom. Übersichtsbild. 

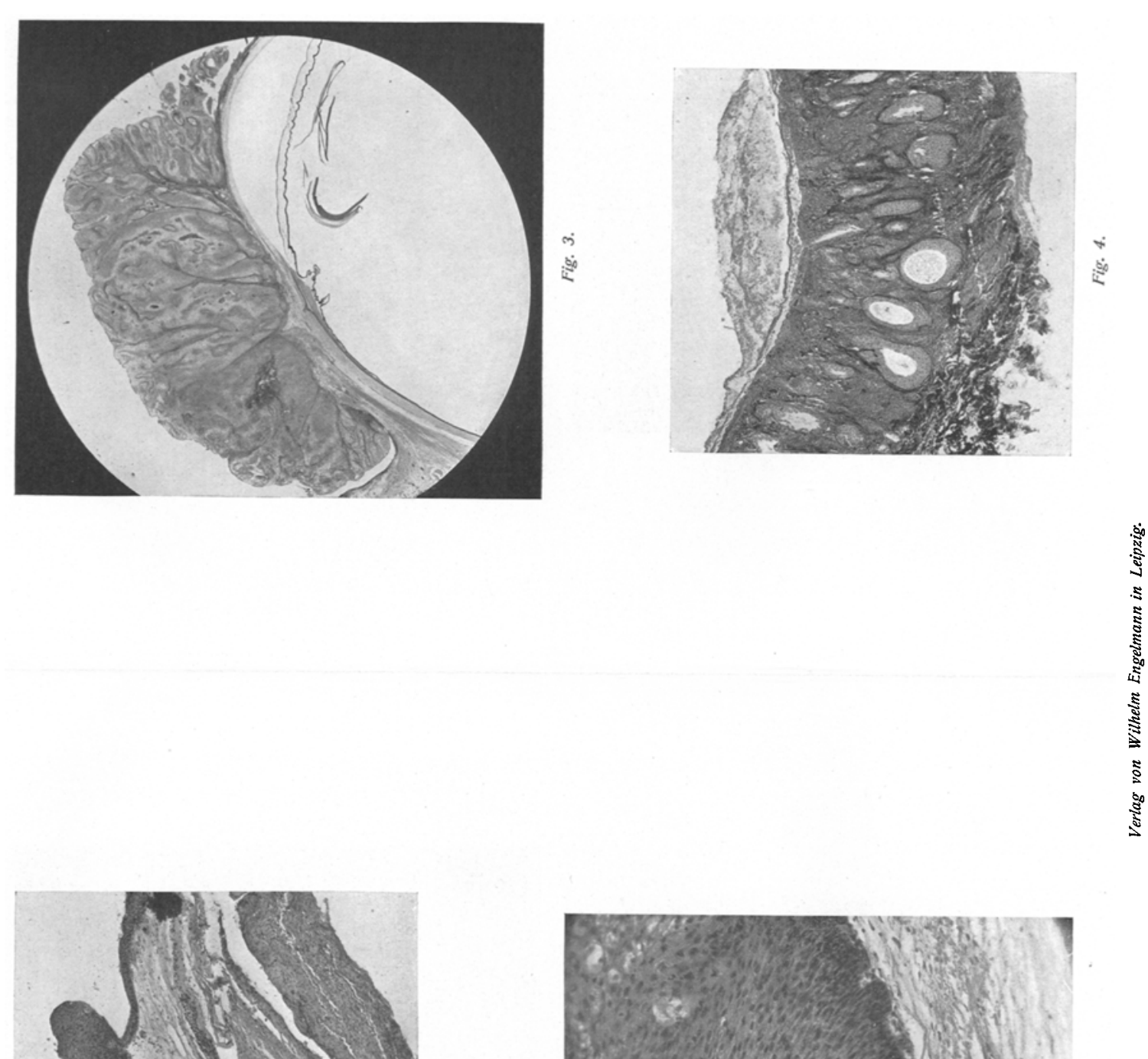


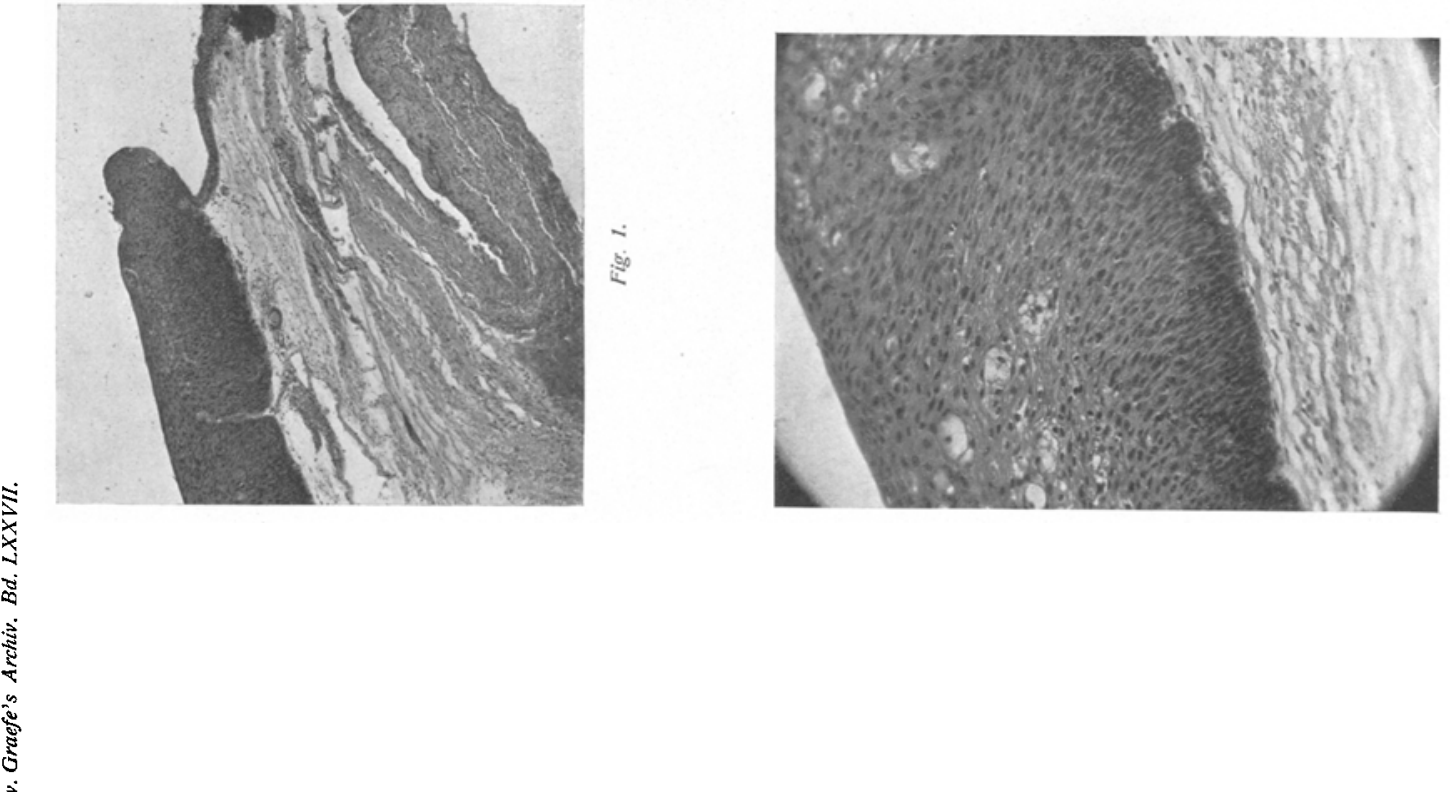